\title{
High speed transmission of signal level for white light emitting diode (LED) as a transmitter device by using modified phase equalization
}

\author{
Maad M. Mijwil \\ Computer Engineering Techniques Department, Baghdad College of Economics Sciences University, Iraq
}

\begin{tabular}{l}
\hline \hline Article Info \\
\hline Article history: \\
Received Jul 11, 2019 \\
Revised Aug 1, 2019 \\
Accepted Sep 25, 2019 \\
\hline
\end{tabular}

Keywords:

Equalization

Visible light communication

White light emitting diode

\begin{abstract}
Visible light communication (VLC) also known as "Li-Fi", uses standard LEDs to transmit data such as information, images, music, and videos. The first LED was developed in 1927 by Oleg Vladimírovich Lósev (19031942), however it was not used in the industry until the 1960s. In this paper, will describe the implementation of Modified Phase Equalization (MPH) on visible white LED lamps signal because it's has slow transition time that severely limits in the communication system data speeds with phase equalization that increases the bandwidth of the LED modulation. Employ two filters with Modified Phase Equalization: first, Complementary (C) filter to combine between all kinds moving signals and second, finite impulse response (FIR) filter to obtain all coefficients that respective. Implementation in two phases: first, frequency $150 \mathrm{KHz}$ with number of signals 15000 signals and second, doubling frequency $300 \mathrm{KHz}$ with number of signals 30000 signals of LEDs without losing their main functionality as illumination sources.
\end{abstract}

Copyright () 2020 Institute of Advanced Engineering and Science. All rights reserved.

\section{Corresponding Author:}

Maad M. Mijwil,

Computer Engineering Techniques Department,

Baghdad College of Economics Sciences University, Iraq.

Email: maadalnaimiy@yahoo.com

\section{INTRODUCTION}

Since the end of the $20^{\text {th }}$ century, a society has changed. Data are becoming increasingly important and symbolic of the digital revolution [1]. Data transmission is one of the cornerstones of development, without the ability to send information back and forth between technical devices, the digital revolution would be unthinkable [2]. The latter has meanwhile covered all areas of life for communication between individuals as well as in entrepreneurial processes [3]. Nowadays, a lot of researchers are working on the development of light-emitting diode (LED) lighting system [4]. The lighting of a home, office or public space can't be used only to generate sensations and have a correct visibility of the space, but also for the transmission of data. This is possible thanks to the technology called Visible Light Communication (VLC), which uses light emitted by an LED to exchange thousands of data to computers, cell phones or tablets. Meanwhile, we encounter LEDs everywhere, no matter if traffic lights, television or lighting in private and business areas. Due to their long life, their small size and the variety of shapes and color spectrums, LEDs have prevailed in a short time compared to light bulbs, halogen and energy-saving lamps [5].

White LEDs are considered as a major candidate for future illumination [6]. Due to the potential for simultaneous use of these light sources for lighting and communication, visible-light communications have been a subject of increasing research and development activities [7]. Light-emitting diodes (LEDs) consist of semiconductor crystal layers depending on the material which is used, the wavelengths of light emitted when a current flow through in one direction, called the forward direction (electroluminescence) [8]. The light up of an individual diode either red, green or blue. White light can be obtained from this, by a various way [9]. 
In the past, light-emitting diodes have mainly served as colorful but dimly lit signal lamps [10]. After decades of research and development, the digitally controllable, energy-saving light bulbs are revolutionizing lighting by replacing obsolete, inefficient light bulbs [11].

The existing problems in implementing any VLC system and it's necessary to take the consider the following limitations: The LED signal as transmitter has a slow switching speed, which limits the transmission speed of the system [12]. The light information which transmitted from the LED will be weakened (fading) while transmitted on the free space channel, it means the farther the distance of the receiver, the weaker the signal received, and the information may not be received at all [13]. The receiver is illuminated by energy sources such as ambient light, natural sunlight, fluorescent lamp light and incandescent lamp light, these sources cause variation in the received photocurrent that is not related to the transmitted signal, which results in an additive noise component in the receiver [14]. The distance of the maximum link is limited by the power of the light source.

In this paper, implemented Phase Equalization with modified which it's used between the transmitter such as white LEDs and the receiver such as computers, cell phones or tablets etc., because it's allowed to increase the bandwidth of modulation of the LED, that achieves the recovery of the sent signal that attenuates from the passage to the transmission channel, using equations of two filters they are Complementary filter and Finite Impulse Response filter with the equalization, makes it easy to adjust to changes between the transmitter and the receiver by modifying the coefficients of this filters.

The rest of the paper is organized as follows: In Section 2, describe System Model. Section 3, Modified Phase Equalization and Results. Performance Evaluation is discussed in Section 4. Conclusions is presented in Section 5.

\section{SYTEM MODULE}

VLC is considered a futuristic technology applied to both data communication and illumination [15], VLC broadcasting is limited to the application to the Internet services, where there are various applications and all receivers can communicate within the area of illumination sources [16]. VLC has several implementation challenges, one of the biggest challenges is improving the transmission speed of data with high spectral efficiency [17]. An optical links is a telecommunications links, intensity modulation techniques (IM) are used with direct detection (DD) is popular techniques in optical communication, being a viable transmission technique in indoor links [18]. A waveform with the information to be transmitted on VLC links by directly modulates of the instantaneous optical power emitted by an LED through the propagation channel and this information is retrieved by the receiver in end by direct detection on a photodiode [19] as shown in the Figure 1 [20].

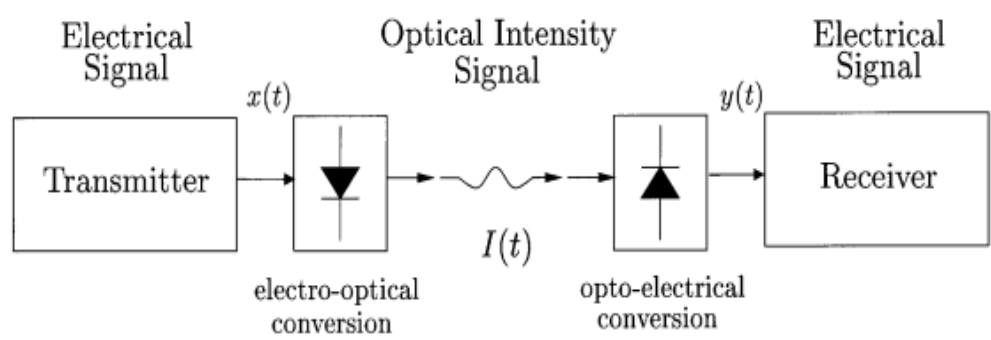

Figure 1. A simplified block diagram of an optical intensity direct detection communications system

An Optical wireless communication system (OWCS) can be generated by an equivalent baseband system is a signal that has a near-zero frequency range [21] as shown in Figure 2 [22], where $X(t)$ is the time signal that the transmitted signal suffers, as a consequence of the propagation channel, is modelled by an impulse response $\mathrm{Rh}(\mathrm{t})$, where $\mathrm{R}$ is a coefficient which symbolized the photodiode response and $\mathrm{h}(\mathrm{t})$ is the impulse response function (IRF) of the linear time-invariant (LTI) [23]. The noises that generates of the ambient light is looks like as white noise, Additive Color, Gaussian noise and independent of the signal be transmitted [24]. This additive residue has been represented as $\mathrm{N}(\mathrm{t})$ [25]. 


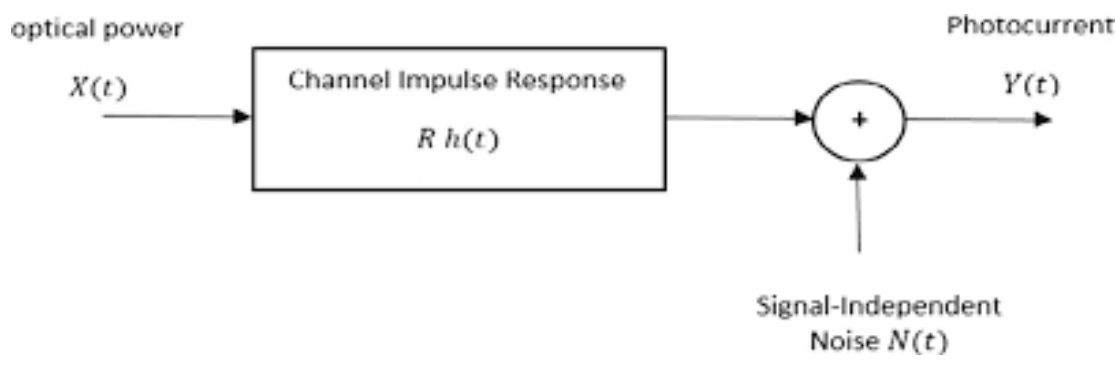

Figure 2. Effects of selecting different switching under dynamic condition

This allows characterizing a VLC link by the equation [26]:

$$
Y(t)=X(t) \otimes R h(t)+N(t)
$$

where $\otimes$ denotes convolution

\section{MODIFIED PHASE EQUALIZATION AND RESULT}

Equalization generally processes the overall frequency response of the electrical signal with a frequency filter. In this work, it's has been applied to a number of signals $N$ between 15000 and 30000 signal from LEDs at a frequency $f s=150 \mathrm{KHz}$ with time $T=1 / f s$ with values determined of coefficients $\operatorname{Cof}$ for the frequency response of desired $G_{(z)}$ with $N$ and also only one coefficient $A$ for $\operatorname{hled}_{(z)}$ with $N$ where $z$ is values of coefficients, number of signals and frequency as shown below:

$$
\begin{aligned}
& \operatorname{hled}_{(z)}=(A, 1, N, f s) \\
& G_{(z)}=(\operatorname{Cof}, 1, N, f s)
\end{aligned}
$$

Creates module functions for Hled, $G_{(z)}, d B$ from the following equations:

$$
\begin{aligned}
& \text { moduleHled }=a b s(\text { hled }(z)) \\
& \text { moduled } B=10 * \log _{10}(\text { moduleHled })
\end{aligned}
$$

where $\mathrm{dB}$ is a decibel is a unit used to measure the intensity of sound and also used in other specialties in acoustics, electricity and telecommunications.

$$
\begin{aligned}
& \operatorname{module}_{(z)}=\operatorname{abs}\left(G_{(z)}\right) \\
& \text { module }_{(z)} d B=20 * \log _{10}\left(\text { module }_{(z)}\right) * T
\end{aligned}
$$

The implementation as shown in Figure 3 when used $N=15000$ signals at $f s=150 \mathrm{KHz}$ where the results of desired frequency response $G_{(z)}$, which are in red color in the one direction at value of $0 \mathrm{~dB}$, while frequency response of $\operatorname{Hled}_{(z)}$, which are in blue color also in one direction at a value of $9 \mathrm{~dB}$. The another implementation as shown in Figure 4 when used $N=30000$ signals at $f s=300 \mathrm{KHz}$ it is affected by the change in the number of signals or frequency, hat it is required to changing frequency response from LEDs and desired frequency response $G_{(z)}$ thus leading to an increase the bandwidth of LED and both of them $\operatorname{Hled}_{(z)}$ and $G_{(z)}$ have different moving signals where desired frequency response $G_{(z)}$ is slow moving signals while frequency response from LEDs are fast moving signals. 


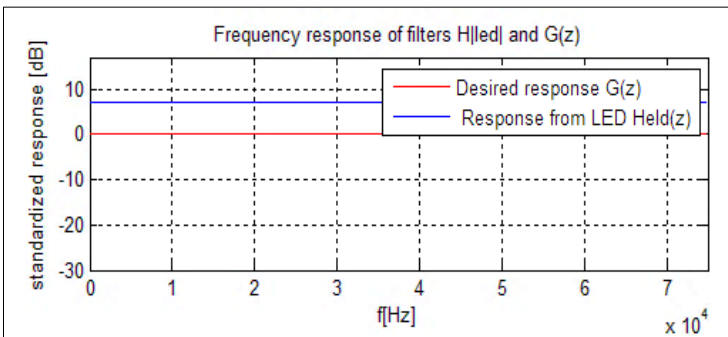

Figure 3. Desired frequency response of $G_{(z)}$ in red color and frequency response of $\mathrm{Hled}_{(z)}$ in blue color with $N=15000$ signals at $f s=150 \mathrm{KHz}$

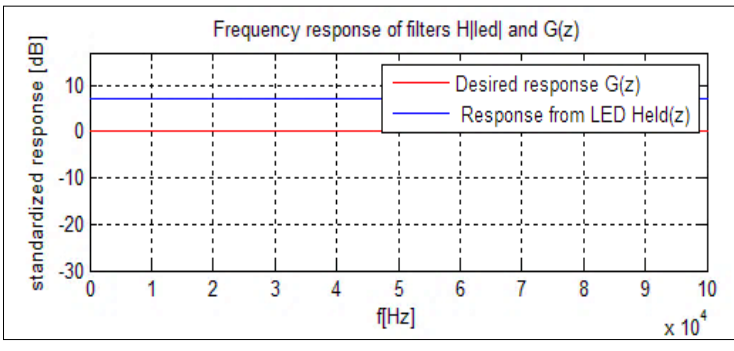

Figure 4. Desired frequency response of $G_{(z)}$ in red color and frequency response of Hled $_{(z)}$ in blue color with $N=30000$ signals at $f s=300 \mathrm{KHz}$ and different $\mathrm{x}$-axis

To achieve a compensation of LED response $\operatorname{Hled}_{(z)}$ by employing zero forcing equalizer (ZFE) which are applied to the inverse of the frequency response of LED, the LED transfer function that must be multiplied by its inverse by ZFE according to the following (7).

$$
C_{(z)=} \frac{1}{\operatorname{Hled}_{(z)}}
$$

The filter resulting from the inverse of the LED transfer function is an ideal filter, which requiring a great digital processing. Due to the indicated a frequency response is analyzed to increase the modulation bandwidth of the LED moduleHled and it's feasible to implement, then to design the complementary filter $C_{(z)}$ the desired frequency response $G_{(z)}$ divided over the frequency response of Hled $(z)$, that can be expressed mathematically by the (8).

$$
\operatorname{module}_{(z)}=\frac{\text { moduleG }_{(z)}}{\text { moduleHled }_{(z)}}
$$

Complementary filter that is used to combine between slow moving signals $G_{(z)}$ and fast moving signals $\operatorname{Hled}_{(z)}$. The result of the division of equation module $_{(z)}$ combine between all signals with different moving as shown in Figure 5 with $N=15000$ signals at $f s=150 \mathrm{KHz}$ and also in Figure 6 with $N=30000$ signals at $f s=300 \mathrm{KHz}$ by using a two-dimensional stem plot in Matlab to displays all signals as lines extending from a baseline along the $\mathrm{x}$-axis while a circle whose $\mathrm{y}$-position represents the data value of all signals terminates for each stem. The difference between each figures are x-axis where Figures 6 when doubling the number of signals $N$ and frequency $f s$ increased the bandwidth of LEDs speed transmission.

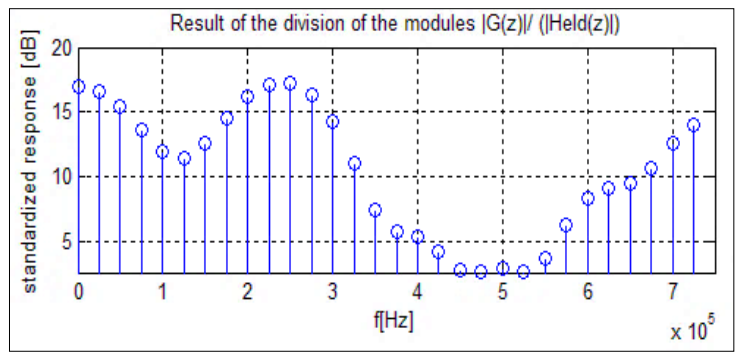

Figure 5. The result of the division of equation module $_{(z)}$ with $N=15000$ signals at

$$
f s=150 \mathrm{KHz}
$$

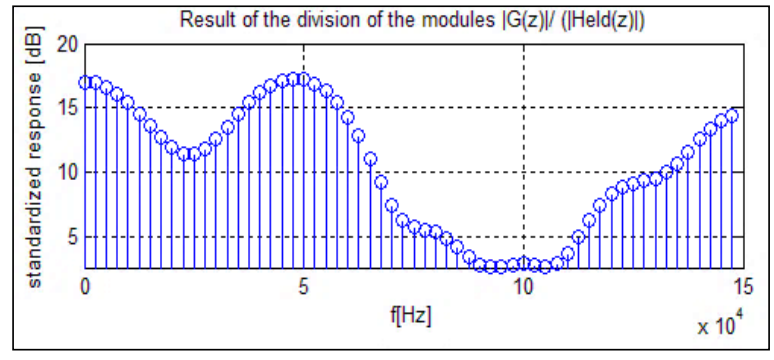

Figure 6. The result of the division of equation module $_{(z)}$ with $N=30000$ signals at $f s=$ $300 \mathrm{KHz}$ and different $\mathrm{x}$-axis

Implementing a finite impulse response (FIR) filter in Matlab with the result of an equation the complementary filter to obtain all coefficients that respective in red color which is a star-shape as shown in Figure 6 it can be analyzed then $C_{(z)}$ is a high pass filter that a compensation value of $17 \mathrm{~dB}$ with $N=15000$ 
at a frequency $f s$ of $150 \mathrm{KHz}$, in Figure 7 and Figure 8 show the result FIR filter when doubling the number of signals and frequency and therefore doubling all coefficients and increasing the modulation bandwidth at the same value in Figure 6 which is $17 \mathrm{~dB}$, in both figures there are no changing in value of complementary filter $C_{(z)}$ no matter how much coefficients values.

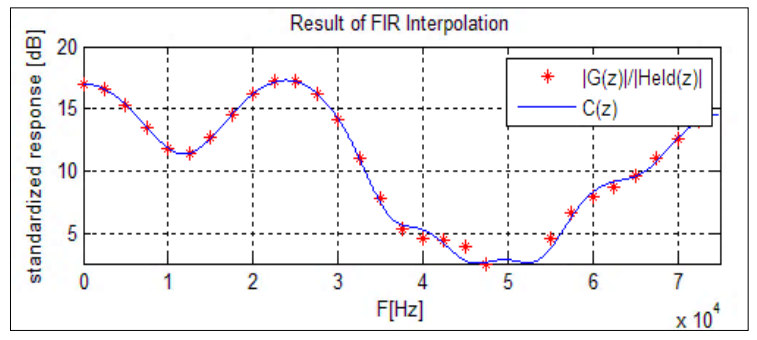

Figure 7. The result of FIR interpolation with $N$ $=15000$ signals at $f s=150 \mathrm{KHz}$

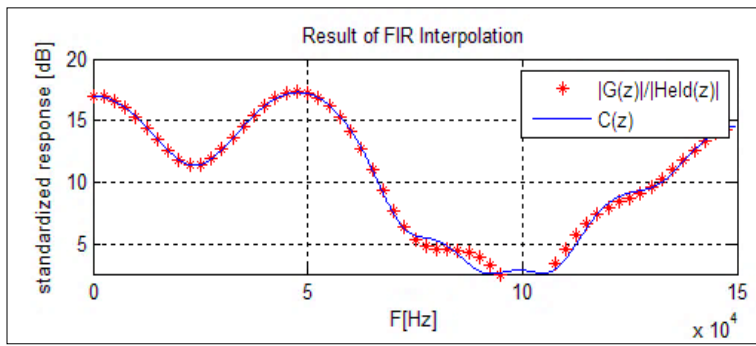

Figure 8. The result of FIR interpolation with $N$ $=30000$ signals at $f s=300 \mathrm{KHz}$ and different $\mathrm{x}$-axis

Creates module functions for FIR interpolation from the following equation:

$$
\begin{aligned}
& h 1=(A, 1, N, f s) \\
& h 2=(B, 1, N, f s) \\
& \bmod H t=20 * \log 10(\operatorname{abs}(h 1 * h 2))
\end{aligned}
$$

where $h 1$ is module Hled while $h 2$ is module FIR interpolation, modHt is the result of module Hled multiply by module FIR interpolation, $N$ is number of signals, $f s$ is frequency and $A, B$ are coefficients of $h 1$ and $h 2$. Figure 9 and Figure 10 shows the difference between all values for $\operatorname{Hled}_{(z)}, C_{(z)}$ and the result of module Hled multiply by module FIR interpolation, where the value of the $\operatorname{Hled}_{(z)}$ in green color remained at value of $0 \mathrm{~dB}$, the signal of $C_{(z)}$ in blue color stay constant at value of $17 \mathrm{~dB}$ while the signal of modHt in red color at value of $3 \mathrm{~dB}$ that is required the signal of LEDs remains constant at a certain value without losing their main functionality as illumination sources.

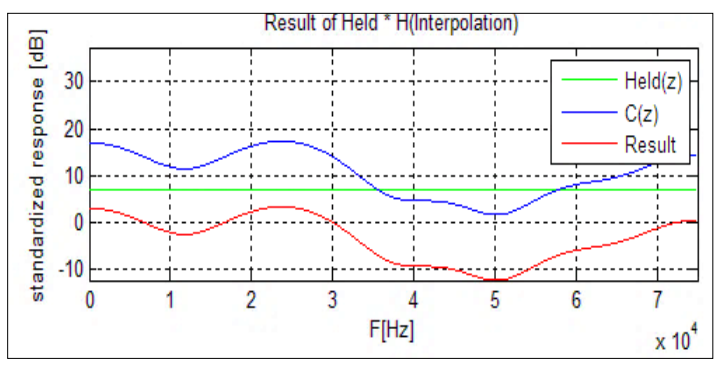

Figure 9. The result of modHt with $N=15000$ signals at $f s=150 \mathrm{KHz}$

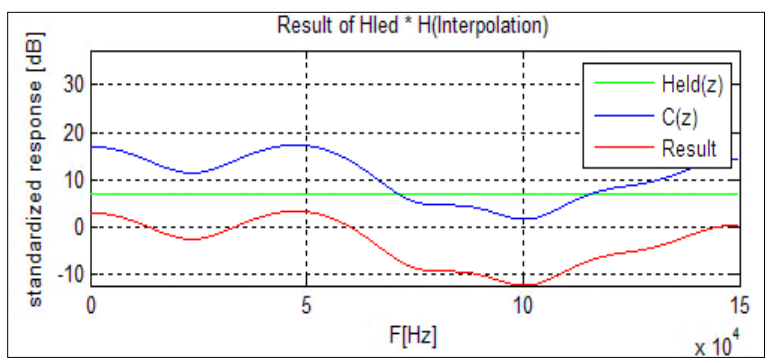

Figure 10. The result of $\operatorname{modHt}$ with $N=30000$ signals at $f s=300 \mathrm{KHz}$ and different $\mathrm{x}$-axis

\section{PERFORMANCE EVALUTION}

The modulation of modified phase equalization (MPH) is created by the Matlab software in this section, a comparison the result bandwidth white LED transmission was made with pre-equalization. From the Figure 11 shows the frequency response of the LED with $N=15000$ signals at $f s=150 \mathrm{KHz}$ at a value of $4 \mathrm{~dB}$, which is the result of the modulation of modified phase equalization (MPH) after applying two filters they are finite impulse response (FIR) filter and complementary filter, while Figure 12 shows the frequency response of the LED with $N=1200$ signals at $f s=30 \mathrm{KHz}$ at a value of $1 \mathrm{~dB}$, which is the result of the pre-equalization with different coefficients value. 


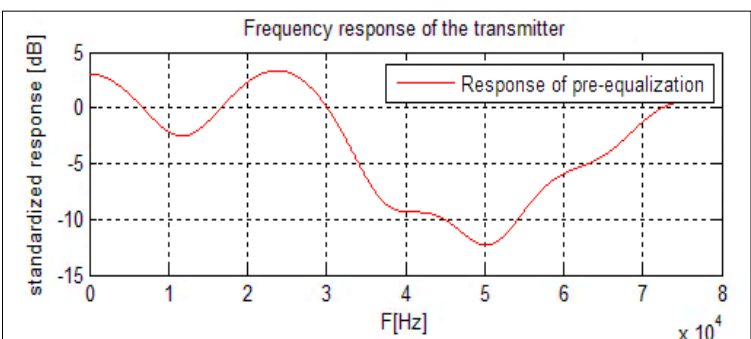

Figure 11. The result of the modulation of modified phase equalization (MPH) with $N=15000$ signals at $f s=150 \mathrm{KHz}$

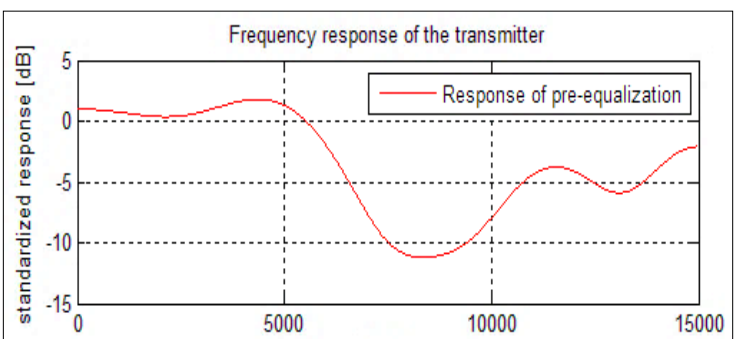

Figure 12. The result of the pre-equalization with $N$ $=1200$ signals at $f s=30 \mathrm{KHz}$

As for the distance of bandwidth of pre-equalization, it's less than from modified phase equalization because they have different number of signals, different frequency and also different coefficient values are explained in both earlier figures with keeping the power of white LEDs unchanged as illumination sources.

\section{CONCLUSION}

Light is a form of energy that has great impact on life on earth no matter if it comes from natural sources such as the sun or artificial sources such as a lamp. Designing the modified phase equalization $(\mathrm{MPH})$ is allowed to improve the modulation of bandwidth white LED between $150 \mathrm{KHz}$ and increasing by $50 \%$ at $300 \mathrm{KHz}$ without introducing any kind of radio whilst the pre-equalization only achieved improved the bandwidth white LED between $15 \mathrm{KHz}$ and also increasing by $50 \%$ at $30 \mathrm{KHz}$. A new coefficients values have proven to be correct values to increase the signal level in high speed data transmission. Complementary filter is combined between slow and fast moving signals and most this signals are fast moving.

\section{REFERENCES}

[1] A. R. Darlis, et al., " Bidirectional Underwater Visible Light Communication," International Journal of Electrical and Computer Enfineering (IJECE), vol. 8, no. 6, pp:5203-5214,Dec 2018.

[2] M. L. R. Rabeh, et al., "Data Transmission via Visible Light Communcation (VLC) Technique," International Journal of Innovative Research in Science,Engineering and Technology, vol. 5,no. 9, pp:16473-16481,Sep 2016.

[3] S. Tarannum, " Data Transmission Through Smart Illumination via " Visible Light Communication Technology"," International Journal of Technical and Applications, vol. 4, no. 2, pp:136-141, Apr 2016.

[4] S. Rajan, et al., "Visible Light Communication,"International Journal of Compuuter Trends and Technology(IJCTT), vol. 4, no.4, pp. 906-910, Apr 2013.

[5] P. Tomi, et al., "Advantages of Wgite LED Lamps and New detctor Technology in Photometry,"Light:Science and Application, vol. 4, pp. 1-7, May 2015.

[6] O. C. Dominic, et al., "Visible Light Communications:Challenges and Possibilities," IEEE $19^{\text {th }}$ International Symposium on Personal, Indoor and Mobile Radio Communications, pp. 1-5, Dec 2008.

[7] S. C. Subhash, " Basics of Light Emitting diodes, Characterizations and Applications,"chapter, Dec 2009.

[8] M. Subramanian, et al., " Red, Green, and Blue LEDs for White Light Illumination," IEEE Journal on Selected Topics in Quantum Electronics, vol. 8, no.2, pp. 333-338, Apr 2002.

[9] Z. Shi, et al., "The implementation of LED color constancy algorithms based on FPGA," IEEE International Conference on Anti-Counterfeiting, Security and Identification (ASID), vol. 10, pp. 1-5, Oct 2013.

[10] C. Janssen, et al., "Opinion on Mercury in Certain Energy-saving Light Bulbs, "European Commission DG SANCO, vol. 7, pp. 1-17, May 2010.

[11] E. Hany, et al., "An LED Model for Intensity-Modulated Optical Communication Systems," IEEE Photonics Technology Letters, vol. 22, no.11, pp. 835-837, Apr 2010.

[12] F. Syifaul, et al., " Analysis of Received Power Characteristics of Commercial Photodiodes in Indoor Los Channel Visible Light Communication, "International Journal of Advanced Computer Science and Applications (IJACSA), vol. 8, no.7, pp. 164-172, Aug 2017.

[13] M. S. M. Gismalla, et al., "Performance Evaluation of Optical Attocells Configuration in an Indoor Visible Light Communication," Indonesian Journal of Electrical Engineering and Computer Science, vol. 14, no.2, pp. 668-676, May 2019.

[14] J. S. Lee, et al., " An LED-Based Visible Light Communication System for Multicast, Indonesian Journal of Electrical Engineering and Computer Science, vol. 13, no.1, pp. 265-271, Jun 2019.

[15] N. B. M. Nawawi, et al., "Performance Evaluation of VLC System Using New Modulation Approach," Bulletin of Electrical Engineering and Informatics, vol. 8, no.2, pp. 630-636, Jun 2019. 
[16] C. Kottke, et al., " Performance of Bandwidth Extension Techniques for High-Speed Short-Range IM/DD Links," Journal of Lightwave Technology, vol. 37, no.2, pp. 665-672, Jun 2019.

[17] E. Basar, et al., "Index Modulation Techniques for Next-Generation Wireless Networks," IEEE Access, vol. 5, pp. 1-51, Aug 2017.

[18] L. Hao, et al., " Indoor Gigabit optical wireless communications: Challenges and possibilities, "12 ${ }^{\text {th }}$ International Conference on Transparent Optical Networks, 2010, pp. 1-6, Aug 2010.

[19] T. Adiono, et al., "Noise and Bandwidth Consideration in Designing Op-Amp Based Transimpedance Amplifier for VLC," Bulletin of Electrical Engineering and Informatics, vol. 7, no.2, pp. 314-322, Jun 2018.

[20] M. Mohammed, et al., "Visible Light Communications Using OFDM and Multiple LEDs," IEEE Transactions on Communications, vol. 63, no.11, pp. 4304-4313, Nov 2015.

[21] H. Hranilovic, et al., " Optical Intensity-Modulated Direct Detection Channels: Signal Space and Lattice Codes," IEEE Transactions on Information Theory, vol. 49, no.6, pp. 1385-1399, Jun 2005.

[22] G. Itziar, et al., "Discrete Indoor Three-Dimensional Localization System Based on Neural Networks Using Visible Light Communication," Available: https://www.mdpi.com/journal/sensors, vol. 18, no.4, pp. 1-17, Mar 2018.

[23] H. Schulze, et al., " Frequency-Domain Simulation of the Indoor Wireless Optical Communication Channel," IEEE Transactions on Communications, vol. 64, no.6, pp. 1-15, Jun 2016.

[24] W. C. W. Lim, et al., "Wireless Water usage Monitoring System for Home/Small Premises," Indonesian Journal of Electrical Engineering and Computer Science, vol. 15, no. 2, pp:704-713, Aug 2013.

[25] G. M. Aji, et al., " Electronic Information Board Based on AVR Atmega 128 for TEWS in Cilacap," Bulletin of Electrical Engineering and Informatics, vol. 7, no.2, pp:216-221, Jun 2018.

[26] B. C. Jeffrey, "Wireless Infrared Communications," Wiley Encyclopedia of Telecommunications, Preprint, 2002.

\section{BIOGRAPHIES OF AUTHOR}

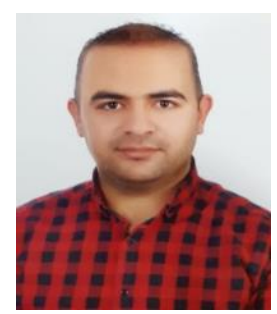

Maad M. Mijwil received B.Sc. degree in Software Engineering from Software Engineering Department at Baghdad College of Economics Sciences University, Iraq in 2008/2009 and M.Sc. degree in Wireless sensor network of computer science from University of Baghdad, Iraq in 2014/2015. Currently, he is working Assistant Lecturer in Baghdad College of Economics Sciences University. His research interests include Genetic Algorithm, Optimization, Artificial Intelligence, Matlab simulation, Machine Learning, Wireless Sensor Networks and Visible Light Communication. 